\title{
A critical evaluation of bioimpedance spectroscopy analysis in estimating body composition during GH treatment: comparison with bromide dilution and dual X-ray absorptiometry
}

\author{
Vita Birzniece ${ }^{1,2,3}$, Chong-Hui Khaw ${ }^{1}$, Anne E Nelson ${ }^{1}$, Udo Meinhardt ${ }^{1,4}$ and \\ Ken K Y Ho ${ }^{1,2,5}$ \\ ${ }^{1}$ Garvan Institute of Medical Research and Department of Endocrinology, St Vincent's Hospital, Sydney, New South \\ Wales, Australia, ${ }^{2}$ The University of NSW, Sydney, New South Wales, Australia, ${ }^{3}$ School of Medicine, University of \\ Western Sydney, Sydney, New South Wales, Australia, ${ }^{4}$ Centre for Pediatric Endocrinology, Zurich, Switzerland and \\ ${ }^{5}$ Centres for Health Research, Princess Alexandra Hospital, Brisbane, Queensland, Australia
}

\author{
Correspondence \\ should be addressed \\ to $\mathrm{K} \mathrm{KY} \mathrm{Ho}$ \\ Email \\ k.ho@uq.edu.au
}

\begin{abstract}
Objective: To compare estimates by bioimpedance spectroscopy analysis (BIS) of extracellular water (ECW), fat mass (FM), and fat-free mass (FFM) against standard techniques of bromide dilution and dual energy X-ray absorptiometry (DXA) during intervention that causes significant changes in water compartments and body composition.

Methods: Body composition analysis using BIS, bromide dilution, and DXA was performed in 71 healthy recreational athletes (43 men, 28 women; aged 18-40 years; BMI $24 \pm 0.4 \mathrm{~kg} / \mathrm{m}^{2}$ ) who participated in a double-blinded, randomized, placebocontrolled study of GH and testosterone treatment. The comparison of BIS with bromide dilution and DXA was analyzed using linear regression and the Bland-Altman method.

Results: At baseline, there was a significant correlation between BIS and bromide dilution-derived estimates for ECW, and DXA for FM and FFM $(P<0.001)$. ECW by BIS was $3.5 \pm 8.1 \%$ lower compared with bromide dilution, while FM was $22.4 \pm 26.8 \%$ lower and FFM $13.7 \pm 7.5 \%$ higher compared with DXA $(P<0.01)$. During treatment, the change in ECW was similar between BIS and bromide dilution, whereas BIS gave a significantly greater reduction in FM $(19.4 \pm 44.8 \%)$ and a greater increase in FFM $(5.6 \pm 3.0 \%)$ compared with DXA $(P<0.01)$. Significant differences in body composition estimates between the BIS and DXA were observed only in men, particularly during the treatment that caused greatest change in water compartments and body composition.
\end{abstract}

Conclusion: In healthy adults, bioimpedance spectroscopy is an acceptable tool for measuring ECW; however, BIS overestimates FFM and substantially underestimates FM compared with DXA.

\section{Introduction}

Measurement of body composition is central to many aspects of patient care and can be assessed by several methods, with dual energy X-ray absorptiometry (DXA) considered the common reference method (1). The classical three-compartment model of body composition consists of fat mass (FM), fat-free mass (FFM), and bone minerals (2). The FFM can be then divided further into extracellular water (ECW) and a functional cellular compartment predominantly composed of muscle, the body cell mass (BCM). Thus, measuring ECW is of major importance in assessing treatment effect on (c) 2015 European Society of Endocrinology Printed in Great Britain
Published by Bioscientifica Ltd. 
muscle cell mass. DXA is a valid and reliable method for measuring FM and FFM, whereas for the assessment of ECW tracer dilution methods usually are used. Bromide dilution is a well-established and validated method for the estimation of ECW in humans $(3,4,5)$. However, use of DXA and bromide dilution is limited due to cost, invasiveness, lack of portability, and the need of trained operators.

Bioelectrical impedance analysis (BIA) has become an increasingly popular alternative for the assessment of body composition due to the relatively inexpensive equipment, portability, ease of use and absence of health risks to volunteers. BIA provides an indirect estimate of ECW and total body water (TBW), from which FFM is determined by the use of hydration constant and FM is then calculated by subtracting FFM from the total body weight (6). The measurements are derived based on resistivity coefficients that are gender specific. Thus, the estimates of FFM and FM by BIA depend on many variables.

Different BIA techniques (single-, multi-frequency, and bioimpedance spectroscopy) can be used for the assessment of body composition. The single-frequency approach in BIA has poor precision of estimates, which is only partly corrected by introducing multiple-frequency BIA technique (7). It has been suggested that bioimpedance spectroscopy analysis (BIS) is more accurate because it uses a spectrum of frequencies and the Cole-Cole model in its estimations (8). A few studies have compared BIS with DXA in the assessment of body composition, showing disagreement between the methods in measuring FM and FFM $(9,10,11,12)$. There is a paucity of data that systematically validates BIS in assessing all aspects of body composition during interventions that causes changes in water compartments, FM, and FFM.

ECW measurements are of central importance in dissecting hormone effects on body composition, in particular on muscle mass, as approximate estimation of the functional compartment of muscle mass can be obtained by subtracting ECW from the FFM. Growth hormone (GH) and testosterone are known for their anabolic effects. However GH, particularly when combined with testosterone administration, results in fluid retention (13). Therefore, during these interventions, measuring FFM by DXA will not accurately reflect changes in functional muscle mass but rather may reflect an increase in ECW content. As BIS may be a convenient, fast, and cost-effective tool to measure ECW, providing also assessment of FFM and FM, we aimed to compare estimates by BIS of ECW, FM and FFM against bromide dilution and DXA by examining the agreement between the methods while assessing GH and testosterone effects in healthy adults in a previously published study (14).

\section{Methods}

\section{Study design}

We performed a double-blinded, randomized, placebocontrolled study of 96 healthy recreationally trained athletes, aged 18-40 years, who were in regular training ( $\geq 2$ sessions per week) for the last 12 months. None of the women received any hormonal birth control medication. The data on body composition assessed by DXA and bromide dilution have been previously published (14). Women $(n=28)$ were randomized into two groups: i) GH $(2 \mathrm{mg} /$ day s.c.; $n=15)$ or ii) placebo $(n=13)$. Men $(n=43)$ were randomized into four treatment groups: i) GH ( $2 \mathrm{mg} /$ day s.c.) plus testosterone $(250 \mathrm{mg} /$ week i.m.; $n=11)$; ii) GH plus placebo testosterone $(n=9)$; iii) testosterone plus placebo GH $(n=11)$; and iv) double placebo $(n=12)$. Body composition assessment with DXA, bromide dilution assay, and BIS were done at baseline (week 0), and during treatment (week 8), and these measurements were done on the same day. Due to equipment availability, BIS measurements were undertaken only on 53 subjects (female $=22$, male $=31$ ) at baseline and 71 subjects (female $=28$, male $=43$ ) during treatment. The St Vincent's Hospital Human Research Ethics Committee approved the study and all subjects provided written informed consent before participation. The study was registered with the Australian New Zealand Clinical Trials Registry (ACTRN012605000508673).

\section{Height and weight}

Body weight was measured to the nearest $0.1 \mathrm{~kg}$ using an electronic scale (Tanita BWB-600) without shoes. Height was measured to the nearest $0.5 \mathrm{~cm}$ standing without shoes using a stadiometer (Holtain Ltd, Crymmych, Pembs, UK).

\section{Dual energy X-ray absorptiometry}

FM and FFM were measured by DXA (Model DPX, software version 3.1, Lunar Radiation Corp., Madison, WI, USA). The coefficients of variation (CV) for FFM and FM are 1.4 and $2.9 \%$ respectively (15).

\section{Bromide dilution assay}

Serum bromide was measured by HPLC. The serum samples were de-proteinated by centrifugation through a filtration unit with a cut-off size of $10 \mathrm{kDa}$ (Amicon YM10, Millipore Corp., Bedford, MA, USA). The protein-free ultra-filtrate was 
passed through an anion exchange column (IC-Pak A, Waters Corp., Milford, MA, USA) at a flow rate of $0.35 \mathrm{ml} / \mathrm{min}$ and a detection wavelength of $195 \mathrm{~nm}$. ECW was calculated from the change in the serum bromide concentration 140 min after injection of a known amount of bromide using the formula reported by Miller et al. (16): ECW $(\mathrm{l})=0.9 \times 0.95 \times \mathrm{Br}$ dose $(\mathrm{mmol}) / \Delta \mathrm{Br}$ serum $(\mathrm{mmol} / \mathrm{l})$, where $\Delta \mathrm{Br}$ serum is the change in serum bromide concentration, 0.9 is the correction factor for non-extracellular distribution of bromide, and 0.95 is the correction factor for Donnan equilibrium. For ECW inter-assay and intra-assay CV were 1.6 and $0.3 \%$ respectively (14).

\section{Bioimpedance spectroscopy analysis}

Body composition was assessed by BIS using the ImpediMed Ltd model SFB7 analyzer (ImpediMed Ltd, Brisbane, Queensland, Australia). SFB7 is a bioimpedance spectroscopy device that scans 256 frequencies from 4 to $1000 \mathrm{kHz}$. The measurement is based on an assumption that an electric current at low frequencies cannot permeate cells and travels through extra-cellular space only and is used to measure ECW. An electric current at high frequencies permeates cell membranes and is used to measure TBW. BIS estimates body composition using the mathematical model based on a Cole-Cole analysis (6). It uses the Hanai mixture theory, which models the body as a series of cylinders each having specific resistivity and gender-specific resistivity coefficients, which along with height and weight are used to calculate body fluid compartments (17). The following resistivity coefficients were used: pECW 310.0 and 316.2, and pICW 1018.0 and 1023.5 for males and females respectively. FFM is then derived by dividing TBW with the hydration constant 0.732 (FFM $=$ TBW/0.732) (18). FM is estimated by subtracting FFM from the total body weight. Measurements are taken with subjects in supine position after a 5 min rest with their arms by their sides, but separated from their body with their palms down. Two electrodes placed on the dorsal surface of the right hand/wrist, and another two electrodes on the right foot/ankle according to the manufacturer's instructions. Measurements were repeated twice and the average was taken as the measured value. Inter-assay and intra-assay CV for ECW were 2.2 and $0.2 \%$, for FFM 1.8 and $0.3 \%$, and for FM 4.3 and 1.2\% respectively.

\section{Statistical analyses}

Regression analysis was used to determine the level of relative agreement between the different techniques.
Bland-Altman analysis (19) with paired $t$-tests was used to determine the absolute limits of agreement between the body composition parameters assessed by DXA, bromide dilution, and BIS. Data are presented as means with S.D.s, unless otherwise stated. A $P$ value of $<0.05$ was considered significant. Statistical analysis was performed using SPSS Statistics 20 (IBM Corp., Armonk, NY, USA).

\section{Results}

The baseline characteristics are summarized in Table 1.

\section{Extracellular water}

At baseline, there was a significant correlation between the bromide dilution assay and BIS for measuring ECW $\left(r^{2}=0.84 ; P<0.001\right)$. When compared with bromide dilution, ECW measured by BIS was significantly lower by $0.7 \pm 1.61(P<0.01$; Fig. $1 \mathrm{~A})$.

During treatment, there was a significant correlation between the bromide dilution assay and BIS for assessing the amount of ECW $\left(r^{2}=0.84 ; P<0.001\right)$. ECW measured by BIS was significantly $(P<0.05)$ lower by $0.6 \pm 2.01$ compared with ECW measured by bromide dilution.

BIS assessment of changes in ECW during treatment showed significant correlation with bromide dilution $\left(r^{2}=0.35 ; P<0.001\right)$. There was no significant difference in assessing changes in ECW between BIS and bromide dilution during treatment with $\mathrm{GH}$, testosterone, or combined hormone administration (Figs $2 \mathrm{~A}$ and $3 \mathrm{~A}$ ). There was no significant gender effect on changes in ECW content during hormone administration when comparing BIS with bromide dilution technique (Fig. 3A).

Table 1 Baseline characteristics of subjects. Data are presented as means \pm S.E.M.

\begin{tabular}{|c|c|c|}
\hline Variable & Women $(n=28)$ & Men $(n=43)$ \\
\hline Age (years) & $29.4 \pm 1.2$ & $27.1 \pm 0.8$ \\
\hline Height $(\mathrm{cm})$ & $167.4 \pm 1.2$ & $181.9 \pm 1.0$ \\
\hline Weight (kg) & $64.0 \pm 2.0$ & $82.8 \pm 2.5$ \\
\hline BMI $\left(\mathrm{kg} / \mathrm{m}^{2}\right)$ & $22.8 \pm 0.6$ & $24.9 \pm 0.6$ \\
\hline IGF1 ( $\mu \mathrm{g} / \mathrm{l})$ & $124.8 \pm 6.9$ & $114.9 \pm 6.4$ \\
\hline $\begin{array}{l}\text { Testosterone } \\
(\mathrm{nmol} / \mathrm{l})\end{array}$ & $1.3 \pm 0.1$ & $23.4 \pm 1.1$ \\
\hline $\begin{array}{l}\text { ECW (I) by bromide } \\
\text { dilution }\end{array}$ & $15.6 \pm 0.3$ & $21.8 \pm 0.6$ \\
\hline $\begin{array}{l}\text { Fat-free mass }(\mathrm{kg}) \\
\text { by DXA }\end{array}$ & $42.3 \pm 0.9$ & $64.0 \pm 1.3$ \\
\hline $\begin{array}{l}\text { Fat mass }(\mathrm{kg}) \\
\text { by } \mathrm{DXA}\end{array}$ & $18.5 \pm 1.3$ & $15.1 \pm 1.3$ \\
\hline
\end{tabular}




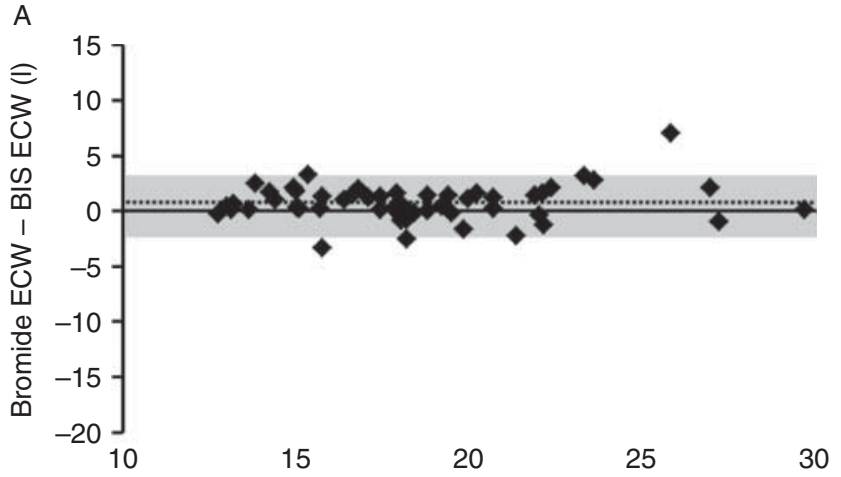

Average of bromide ECW and BIS ECW (I)

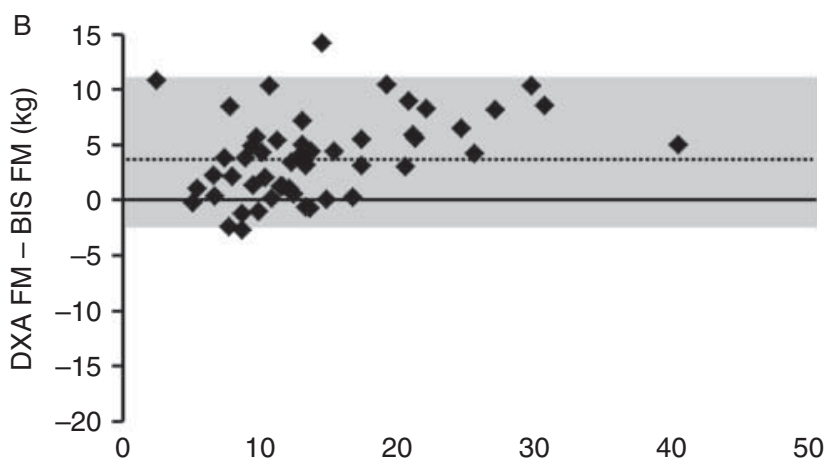

Average of DXA FM and BIS FM $(\mathrm{kg})$

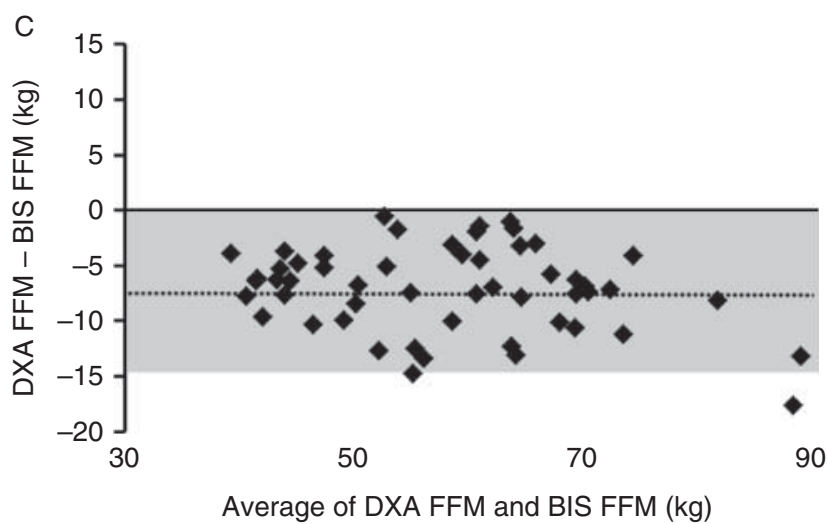

Figure 1

Bland-Altman plots comparing baseline ECW (A), FM (B), and FFM (C) by BIS with bromide dilution (ECW) and DXA (FM and FFM). The dotted line represents mean difference between the methods. The shaded area represents the upper and lower limit of agreement ( \pm 1.96 s.D.).

Thus, when compared with the reference method, BIS underestimated absolute levels of ECW by $3.5 \pm 8.1 \%$ at baseline, whereas the change in ECW during treatment was similar when measured by either BIS or bromide dilution.

\section{Fat mass}

At baseline, there was a significant correlation between DXA and BIS for estimating FM $\left(r^{2}=0.79 ; P<0.001\right)$. When compared with DXA, FM measured by BIS was significantly lower by $3.9 \pm 3.7 \mathrm{~kg}(P<0.001$; Fig. 1B).
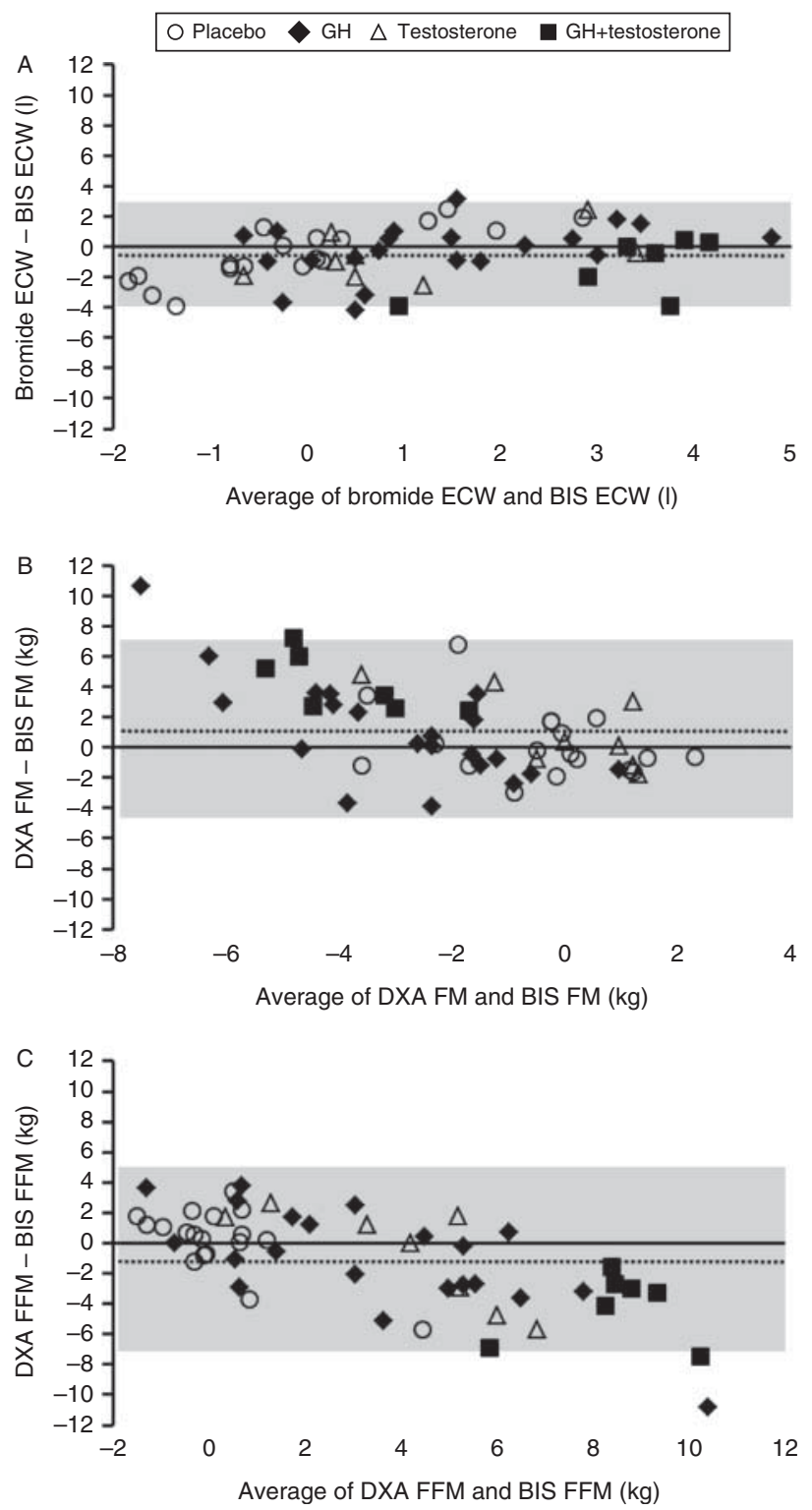

\section{Figure 2}

Bland-Altman plots comparing mean changes in ECW (A), FM (B), and FFM (C) during the treatment measured by BIS, bromide dilution (ECW), and DXA (FM and FFM). The dotted line represents mean difference between the methods in assessing change in ECW, FM, and FFM during GH, testosterone, combined hormone, or placebo administration. The shaded area represents the upper and lower limit of agreement ( \pm 1.96 s.D.). 

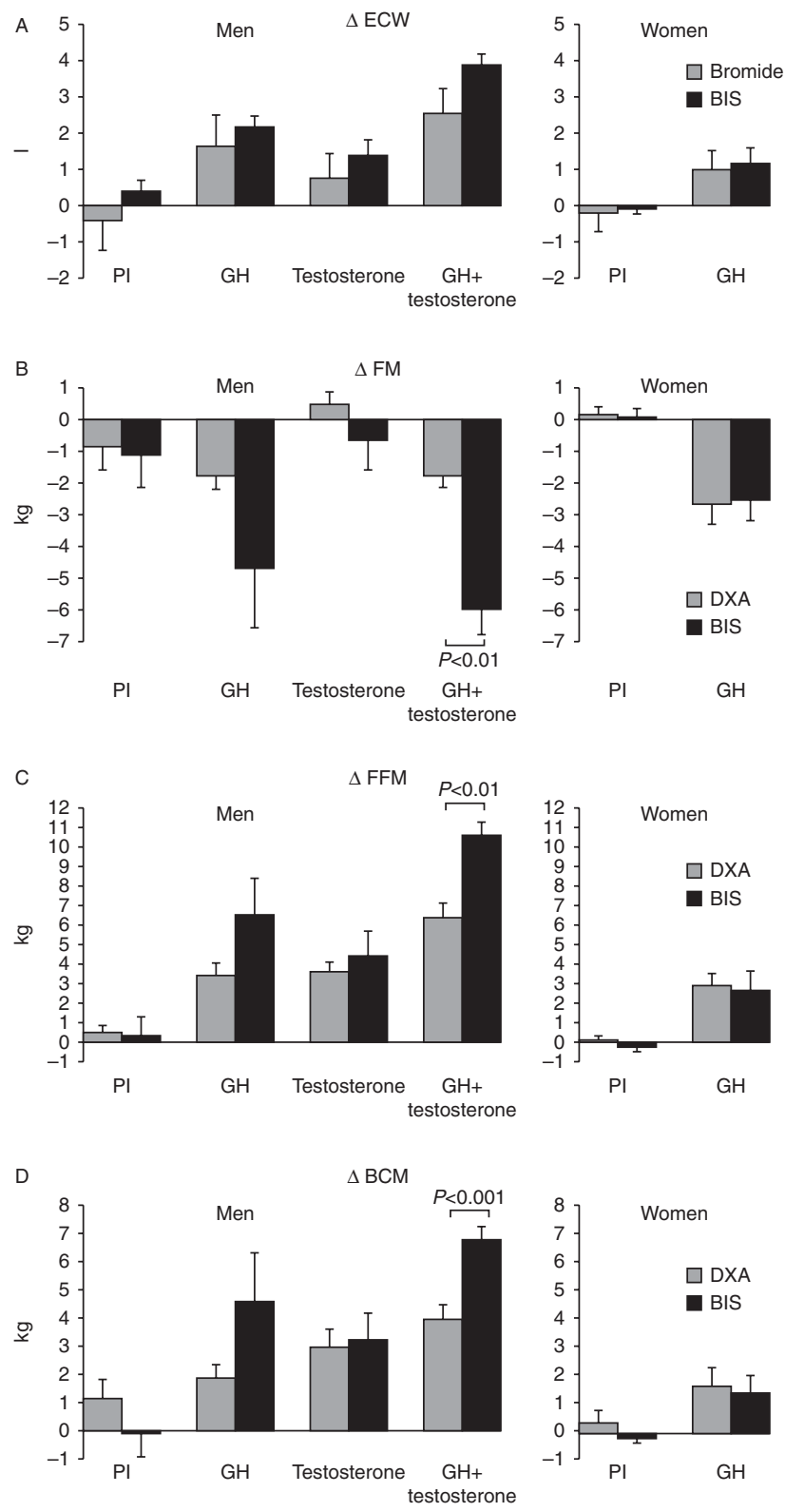

Figure 3

Mean changes in ECW (A), FM (B), FFM (C), and BCM (D) during the treatment with $\mathrm{GH}$, testosterone, combined hormone, or placebo administration. Grey bars represent measurements by bromide dilution or DXA, black bars represent measurements by BIS. Data are presented as means with SEM. BCM, body cell mass; PI, placebo; GH, growth hormone.

During $\mathrm{GH}$ and testosterone administration, FM estimated by BIS significantly correlated with DXA $\left(r^{2}=0.69 ; P<0.001\right)$. BIS-derived FM was significantly lower by $5.0 \pm 4.5 \mathrm{~kg}$ compared with that derived by DXA $(P<0.001)$.
Changes in BIS-derived FM during treatment significantly correlated with those derived by DXA $\left(r^{2}=0.24\right.$; $P<0.001)$. However, the change in BIS-derived FM was significantly lower by $1.2 \pm 3.0 \mathrm{~kg}(P<0.01$; Fig. $2 \mathrm{~B})$. When the change in FM was analyzed separately for each of the treatment groups, the difference between the methods was confined to participants receiving $\mathrm{GH}$, particularly when $\mathrm{GH}$ was combined with testosterone, which was highly significant $(P=0.001$; Fig. 3B). Moreover, this difference was noted in men but not in women (Fig. 3B).

Overall, when compared with DXA, BIS estimate of FM was consistently lower by $22.4 \pm 26.8 \%$ at baseline, and by $40.6 \pm 47.9 \%$ during treatment. Moreover, the measured reduction in FM during treatment was by 19.4 $\pm 44.8 \%$ greater with BIS. In a subgroup of male participants receiving $\mathrm{GH}$ and testosterone-combined administration, the reduction in FM was $71.4 \pm 46.1 \%$ greater as estimated by BIS than by DXA.

\section{Fat-free mass}

At baseline, there was a significant correlation between DXA and BIS for estimating FFM $\left(r^{2}=0.91 ; P<0.001\right)$. However, BIS-derived FFM was by $7.2 \pm 3.8 \mathrm{~kg}$ higher than that measured with DXA $(P<0.001$; Fig. $1 C)$.

During treatment, BIS also significantly correlated with DXA in assessing the amount of FFM $\left(r^{2}=0.91\right.$; $P<0.001)$. When compared with DXA, BIS-derived FFM was significantly higher by $7.9 \pm 4.8 \mathrm{~kg}(P<0.001)$.

Changes in BIS-derived FFM during the treatment showed significant correlation with those derived by DXA $\left(r^{2}=0.6 ; P<0.001\right)$. When compared with DXA, there was a significantly greater increase by $1.0 \pm 3.1 \mathrm{~kg}$ in BIS-derived FFM $(P<0.05$; Fig. 2C). Assessing each treatment subgroup, the overestimation of changes in FFM were significant only in participants receiving combined $\mathrm{GH}$ and testosterone administration $(P<0.01$; Fig. 3C). As with FM, the difference between the methods in assessing FFM was noted only in men but not in women (Fig. 3C). The difference between the methods correlated significantly with the change in FFM during the treatment $\left(r^{2}=0.11 ; P=0.015\right)$, reflecting greater overestimation of FFM by BIS in subjects with the highest increase in FFM.

We next calculated BCM, a functional cellular compartment within FFM, which is derived by subtracting ECW from the FFM. As with FFM, there was a significantly greater increase in BCM measured by BIS compared with the reference methods (Fig. 3D). The difference between the methods in assessing BCM was noted only in men, in 
whom during combined GH and testosterone administration BIS overestimated change in BCM by $2.8 \pm 1.2 \mathrm{~kg}$ $(P<0.001$; Fig. 3D).

Overall, when compared with DXA, BIS estimate of FFM at baseline was $13.7 \pm 7.5 \%$ higher and during the treatment was $13.8 \pm 7.6 \%$ higher. The change in FFM was significantly different between the methods only in men, overestimating the change in FFM by $5.6 \pm 3.0 \%$ and in $\mathrm{BCM}$ by $5.0 \pm 2.6 \%$ during combined $\mathrm{GH}$ and testosterone administration.

\section{Discussion}

In this study of body composition, BIS-derived estimates of ECW, FM, and FFM correlated significantly with those obtained by bromide dilution and DXA in healthy young adults. However, BIS-derived measurements of ECW and FM were significantly lower and FFM significantly higher compared with the reference methods. Treatment with $\mathrm{GH}$, testosterone, or both increased ECW and FFM and reduced FM. BIS-derived measurements of increase in ECW were similar to that estimated by bromide dilution. However, BIS recorded a significantly greater reduction in FM and a greater increase in FFM compared with DXA. These treatment differences in FM and FFM between methods were evident in men but not in women.

BIS utilizes a spectrum of frequencies between 4 and $1000 \mathrm{kHz}$, and employs Hanai mixture conductivity theory and the Cole-Cole model in the estimation of body fluid compartments, which is regarded more accurate than SFBIA or MFBIA $(7,20)$. The estimates are based on assumptions on body shape, tissue density, hydration, and are derived from resistivity coefficients that are gender specific (20). FFM is then estimated by the use of a hydration constant and with FM derived by subtracting FFM from the total body weight (6). Thus, the quantification of body composition by BIS depends on many factors and coefficients that can potentially result in measurement bias. A study in patients with $\mathrm{GH}$ deficiency reported that the bias can be diminished by applying unisex resistivity coefficients derived specifically from this patient population (21). As resistivity coefficients influence measurements by BIS, there is a need to develop coefficients derived from large populations, and incorporating factors, such as age, gender, hydration status, and BMI. Thus, the assumptions and coefficients inherent are likely factors that underlie errors in estimating fluid compartments and body composition by BIS.

This is the first placebo-controlled study comparing BIS with bromide dilution, a classical method for quantifying
ECW, in a healthy population of lean men and women who received GH and/or testosterone administration. Our results show good agreement between the two methods. At baseline, there was only a 0.71 difference in ECW, which represents a $3 \%$ lower estimate by BIS. Importantly, the change in ECW during 8 weeks of GH, testosterone and combined hormone administration showed no significant difference between the methods. Two previous studies evaluated acute shifts in ECW, one involving a hydration/dehydration regimen in healthy men over 4 days and the other in men undergoing surgery. Both these studies reported good agreement between BIS and bromide dilution $(22,23)$. Thus, BIS is a reliable and accurate method for assessing changes in ECW.

In contrast to the good agreement between BIS and bromide dilution in measuring ECW, there was a marked disagreement in FM and FFM estimates between BIS and DXA. At baseline, BIS-derived estimate of FM was $22 \%$ lower and FFM 14\% higher than those obtained by DXA in normal subjects. Previous studies comparing BIA and DXA have also reported similar bias $(9,10,11,12,24)$. Importantly, our study observed that the reduction in FM and increase in FFM during GH and testosterone treatments quantified by BIS substantially differed from that of DXA. The largest discrepancy was for a change in FM, with the reduction in FM almost $20 \%$ greater than by DXA. Collectively, BIS significantly overestimates FFM and underestimates FM compared with DXA.

The observed difference between body composition estimates by BIS and DXA was particularly evident in men receiving $\mathrm{GH}$ alone or in combination with testosterone. These treatments resulted in the greatest increase in FFM, most of which is fluid retention (14). The impact of body composition change in determining BIS accuracy is supported by a weight loss study, which showed greater disagreement between BIS and the reference methods for TBW and ECW measures in patients with the greatest weight loss (25). As the amount of body water determines FFM and FM estimates by BIS, changes in tissue water may be associated with larger discrepancies between BIS and DXA-derived measures of body composition. Moreover, as TBW comprises ECW and ICW, the substantial difference in FFM and FM between the methods in the face of no significant discrepancy between BIS and bromide dilution in ECW estimate may reflect bias in ICW measurements by BIS. Thus, bias in water compartment assessment by BIS may be of the utmost importance in the determination of body composition measurements.

BIS estimates are based on the assumption that TBW comprises $73.2 \%$ of FFM and that this proportion does not 
change. There is a strong evidence that the hydration constant of FFM is not fixed and varies with many factors including the degree of adiposity. For muscle, a major component of the FFM, the average water content is $76 \%$ in normal-weight individuals but only $66 \%$ in obese subjects (26). Thus, the hydration of the FFM is influenced by the degree of adiposity. The increase in hydration derived from increase in TBW in our cohort was $~ 3 \%$ during GH or combined GH and testosterone administration in men. As BIS-derived FFM is estimated by dividing TBW by the hydration constant, an increase in hydration reduces the derived measure of FFM. Assuming that the average TBW in men is 451 in our cohort, an increase in the hydration constant by $3 \%$ from 0.73 to 0.75 reduces FFM by $2.5 \mathrm{~kg}$. Thus, the use of a fixed hydration coefficient introduces a systematic error that is likely to explain in part the overestimation of the FFM by BIS.

Our analysis uncovered interesting gender difference for change in soft tissue composition estimates. We speculate that the gender difference may be related with differences in gender-specific response to GH intervention, resulting in a smaller water retention in women compared with men. As hydration in women would have increased less than in men, it can be predicted that FFM shows better agreement with DXA in women than in men. Genderrelated differences in responsiveness to GH may also introduce additional systematic bias. In our study, the absolute increase in DXA-derived FFM was lower in women than in men (2.7 vs $3.5 \mathrm{~kg}$ ), in whom combined treatment with testosterone further increased FFM $(6.4 \mathrm{~kg})$. Thus, the increase in FFM was far greater in men than in women. As a component of the increase in FFM is ECW, the parallel increase in hydration will lead to a greater estimate of FFM by BIS, as was observed. More studies are required to develop hydration and gender-specific correction factors to improve the accuracy of estimating body composition by BIS in health and disease.

A reason for discrepancies between BIS and DXA may arise from assumptions used in DXA measurement of different tissue compartments. DXA estimates FFM on the basis of greater attenuation of X-rays going through lean tissue compared with fat tissue, not taking tissue water content into account. An increase in weight in parallel with an increase in FFM measured by DXA has been reported after normal saline infusion, reflecting errors in assessing FFM by DXA (27). Moreover, assessment of DXA compared with the four-compartment model has reported bias that varies according to the sex, size, fat amount, and disease state of the subjects, showing that DXA is unreliable for patients who undergo significant changes in body composition $(28,29)$. Thus, DXA is only assumed to be the reference standard method for measuring body composition. Measurement bias by DXA compared with the four-compartment model should be taken into account when comparing accuracy of other methods for body composition measurements, such as BIS, particularly when assessing significant changes in body composition.

In summary, BIS accurately estimates ECW, markedly underestimates FM, and overestimates FFM in healthy young adults. Importantly, when compared with DXA, the reduction in FM and increase in FFM during intervention was significantly greater by BIS. There was a gender difference for changes in FM and FFM, with the highest discrepancy between the methods in men who received combined GH and testosterone administration. This discrepancy may reflect the use of incorrect resistivity coefficients and/or hydration constant for deriving body composition estimates by BIS. The results reflect differences between BIS and the reference methods undertaken in healthy recreational athletes. The findings may be different in hypopitutiary patients.

This is the first study systematically comparing BIS with bromide dilution and DXA in a healthy population of lean men and women undergoing intervention that results in significant changes in tissue water and body composition. We conclude that BIS is an accurate, time and cost-efficient method for estimating ECW; however, it overestimates FFM and substantially underestimates FM compared with DXA.

Declaration of interest

The authors declare that there is no conflict of interest that could be perceived as prejudicing the impartiality of the research reported.

\section{Funding}

This study was supported by funding from The World Anti-Doping Agency and Australian Government Anti-Doping Research Program. The study was registered with the Australian New Zealand Clinical Trials Registry (ACTRN012605000508673).

\section{Acknowledgements}

The authors thank Novo Nordisk for providing Somatropin and Organon for providing Sustanon.

\section{References}

1 Prior BM, Cureton KJ, Modlesky CM, Evans EM, Sloniger MA, Saunders $\mathrm{M} \&$ Lewis RD. In vivo validation of whole body composition 
estimates from dual-energy X-ray absorptiometry. Journal of Applied Physiology 199783 623-630. (doi:10.1007/BF02778056)

2 Heymsfield SB, Wang Z, Baumgartner RN \& Ross R. Human body composition: advances in models and methods. Annual Review of Nutrition 199717 527-558. (doi:10.1126/science.6136091)

3 Vaisman N, Pencharz PB, Koren G \& Johnson JK. Comparison of oral and intravenous administration of sodium bromide for extracellular water measurements. American Journal of Clinical Nutrition 198746 1-4. (doi:10.2527/jas.2011-4684)

4 Cheek DB. Estimation of the bromide space with a modification of Conway's method. Journal of Applied Physiology 19535 639-645.

5 Wong WW, Sheng HP, Morkeberg JC, Kosanovich JL, Clarke LL \& Klein PD. Measurement of extracellular water volume by bromide ion chromatography. American Journal of Clinical Nutrition $1989 \mathbf{5 0}$ 1290-1294. (doi:10.1095/biolreprod66.5.1555)

6 Cornish BH, Ward LC, Thomas BJ, Jebb SA \& Elia M. Evaluation of multiple frequency bioelectrical impedance and Cole-Cole analysis for the assessment of body water volumes in healthy humans. European Journal of Clinical Nutrition 199650 159-164. (doi:10.2527/jas.2014-7808)

7 Gudivaka R, Schoeller DA, Kushner RF \& Bolt MJ. Single- and multifrequency models for bioelectrical impedance analysis of body water compartments. Journal of Applied Physiology 199987 1087-1096. (doi:10.1677/joe.0.1470565)

8 Ayllon D, Seoane F \& Gil-Pita R. Cole equation and parameter estimation from electrical bioimpedance spectroscopy measurements - a comparative study. Conference Proceedings: IEEE Engineering in Medicine and Biology Society 20092009 3779-3782. (doi:10.2337/diabetes.48.4.828)

9 Wang JG, Zhang Y, Chen HE, Li Y, Cheng XG, Xu L, Guo Z, Zhao XS, Sato T, Cao QY et al. Comparison of two bioelectrical impedance analysis devices with dual energy $\mathrm{X}$-ray absorptiometry and magnetic resonance imaging in the estimation of body composition. Journal of Strength and Conditioning Research 201227 236-243. (doi:10.2527/jas.2014-7713)

10 Leahy S, O'Neill C, Sohun R \& Jakeman P. A comparison of dual energy X-ray absorptiometry and bioelectrical impedance analysis to measure total and segmental body composition in healthy young adults. European Journal of Applied Physiology 2012112 589-595. (doi:10.1210/en.2010-1415)

11 Verdich C, Barbe P, Petersen M, Grau K, Ward L, Macdonald I, Sorensen TI \& Oppert JM. Changes in body composition during weight loss in obese subjects in the NUGENOB study: comparison of bioelectrical impedance vs dual-energy X-ray absorptiometry. Diabetes \& Metabolism 201137 222-229. (doi:10.1210/endo-117-6-2435)

12 Eisenkolbl J, Kartasurya M \& Widhalm K. Underestimation of percentage fat mass measured by bioelectrical impedance analysis compared to dual energy X-ray absorptiometry method in obese children. European Journal of Clinical Nutrition 200155 423-429. (doi:10.1159/000109095)

13 Johannsson G, Gibney J, Wolthers T, Leung KC \& Ho KK. Independent and combined effects of testosterone and growth hormone on extracellular water in hypopituitary men. Journal of Clinical Endocrinology and Metabolism 200590 3989-3994. (doi:10.1095/biolreprod60.2.216)

14 Meinhardt U, Nelson A, Hansen J, Birzniece V, Clifford D, Leung K, Graham K \& Ho K. The effects of growth hormone on body composition and physical performance in recreational athletes: a randomized placebo-controlled trial. Annals of Internal Medicine 2010 152 568-577. (doi:10.1677/joe.0.1650519)

15 O'Sullivan AJ, Kelly JJ, Hoffman DM, Freund J \& Ho KK. Body composition and energy expenditure in acromegaly. Journal of Clinical
Endocrinology and Metabolism 199478 381-386. (doi:10.1016/01969781(85)90075-0)

16 Miller ME, Cosgriff JM \& Forbes GB. Bromide space determination using anion-exchange chromatography for measurement of bromide. American Journal of Clinical Nutrition 198950 168-171. (doi:10.1073/ pnas.090099697)

17 Hanai T. Electrical properties of emulsions. In Emulsion Science, ch 5, Ed. P Sherman, London, NY: Academic Press, 1968. doi:10.1046/j.13652826.2003.01087.x.

18 Wang ZM, Pierson RN Jr \& Heymsfield SB. The five-level model: a new approach to organizing body-composition research. American Journal of Clinical Nutrition 199256 19-28.

19 Bland JM \& Altman DG. Statistical methods for assessing agreement between two methods of clinical measurement. Lancet 1986 1307-310.

20 Jaffrin MY \& Morel H. Body fluid volumes measurements by impedance: a review of bioimpedance spectroscopy (BIS) and bioimpedance analysis (BIA) methods. Medical Engineering \& Physics 200830 1257-1269. (doi:10.2527/jas.2005-624)

21 Tengvall M, Ellegard L, Bosaeus N, Isaksson M, Johannsson G \& Bosaeus I. Bioelectrical impedance spectroscopy in growth hormone-deficient adults. Physiological Measurement 201031 59-75. (doi:10.1210/endo-116-1-375)

22 Higgins KJ, Reid PM, Going SB \& Howell WH. Validation of bioimpedance spectroscopy to assess acute changes in hydration status. Medicine and Science in Sports and Exercise 200739 984-990. (doi:10.1210/endo-115-3-1179)

23 Plank LD \& Hill GL. Use of bioimpedance spectroscopy to assess effects of perioperative treatment with growth hormone on fluid changes in patients undergoing major surgery. Annals of the New York Academy of Sciences 2000904 190-192. (doi:10.1016/j.anifeedsci.2003.10.006)

24 Sun G, French CR, Martin GR, Younghusband B, Green RC, Xie YG, Mathews M, Barron JR, Fitzpatrick DG, Gulliver W et al. Comparison of multifrequency bioelectrical impedance analysis with dual-energy X-ray absorptiometry for assessment of percentage body fat in a large, healthy population. American Journal of Clinical Nutrition $2005 \mathbf{8 1}$ 74-78. (doi:10.1126/science.185.4155.949)

25 Cox-Reijven PL, van Kreel B \& Soeters PB. Accuracy of bioelectrical impedance spectroscopy in measuring changes in body composition during severe weight loss. JPEN. Journal of Parenteral and Enteral Nutrition 200226 120-127.

26 Mingrone G, Bertuzzi A, Capristo E, Greco AV, Manco M, Pietrobelli A, Salinari S \& Heymsfield SB. Unreliable use of standard muscle hydration value in obesity. American Journal of Physiology. Endocrinology and Metabolism 2001280 E365-E371.

27 Lands LC, Hornby L, Hohenkerk JM \& Glorieux FH. Accuracy of measurements of small changes in soft-tissue mass by dual-energy X-ray absorptiometry. Clinical and Investigative Medicine 199619 279-285. (doi:10.2527/jas.2005-638)

28 Williams JE, Wells JC, Wilson CM, Haroun D, Lucas A \& Fewtrell MS. Evaluation of Lunar Prodigy dual-energy X-ray absorptiometry for assessing body composition in healthy persons and patients by comparison with the criterion 4-component model. American Journal of Clinical Nutrition 200683 1047-1054. (doi:10.2527/jas.2005-636)

29 Mahon AK, Flynn MG, Iglay HB, Stewart LK, Johnson CA, McFarlin BK \& Campbell WW. Measurement of body composition changes with weight loss in postmenopausal women: comparison of methods. Journal of Nutrition, Health \& Aging 200711 203-213. (doi:10.2527/jas.2005-637)
Received 6 August 2014

Revised version received 24 September 2014 Accepted 17 October 2014 